\section{HIPÓTESE SOBRE O COMPORTAMENTO DO REFLEXO PRESSOR DURANTE O MÉTODO OCLUSÃO VASCULAR EM INDIVÍDUOS COM DOENÇAS CARDIOVASCULARES}

O reflexo pressor que é influenciado pelos estímulos mecânicos e metabólicos que ocorrem nos músculos durante a prática do exercício, medeia o controle sobre a resposta neural, circulatória e também cardíaca. Ele possui dois componentes importantes, os mecanorreflexos e os metaborreflexos musculares (BELLI et al., 2011; SPRANGER et al., 2015).

A importância desses dois componentes durante o exercício é que eles são ativados quimicamente e mecanicamente através de receptores sensíveis localizados no músculo e o método oclusão vascular tem impacto importante sobre esses dois componentes (BELLI et al., 2011; SPRANGER et al., 2015).

Estímulos químicos como o ácido lático, especialmente a acidose são capazes de ativar as fibras aferentes sensoriais IV que são predominantemente associadas aos metaborreflexos. Além disso, estímulos mecânicos como a pressão exercida pela braçadeira durante o método oclusão vascular e alongamento ativam primariamente as fibras aferentes III que são predominantemente associadas aos mecanorreflexos (BELLI et al., 2011; SPRANGER et al., 2015).

No entanto, indivíduos hipertensos, com doença arterial periférica e insuficiência cardíaca congestiva já apresentam aumento do reflexo pressor, sendo exacerbado pela prática do exercício físico realizado juntamente com a oclusão vascular (BELLI et al., 2011; SPRANGER et al., 2015; PIEPOLI et al., 2006; PONIKOWSKI et al., 2011). 
Outra questão importante para se avaliar durante o treinamento com oclusão vascular é a resposta neuro-hormonal envolvida. Resultados de estudos anteriores demonstraram que o exercício físico realizado com oclusão vascular reduziu significativamente a atividade parassimpática, aumentou significativamente a atividade simpática, aumentou significativamente as concentrações de noradrenalina, lactato e hormônio do crescimento quando comparado com o exercício realizado sem oclusão vascular (SHIMIZU e al., 2016; IIDA et al., 2007; IIDA et al., 2005; TAKANO et al., 2005a; TAKANO et al., 2005b). Com isso, demonstra-se que a resposta neuro-hormonal é significativamente superior quando o treinamento é realizado com oclusão vascular.

Ao considerar o efeito do método oclusão vascular sobre o reflexo pressor e comando central com subsequente hiperatividade simpática e o aumento dos riscos adversos relacionados aos problemas cardiovasculares. A segurança do método oclusão vascular deve ser avaliada com cautela antes de sua aplicação (SPRANGER $e t$ al., 2015). A seguir, temos a Tabela 1 adaptada de estudos anteriores (SHIMIZU et al., 2016; SPRANGER et al., 2015; IIDA et al., 2007; IIDA et al., 2005; TAKANO et al., 2005a; TAKANO et al., 2005b) sobre os efeitos neuro-hormonais e cardiovasculares promovidos pelo reflexo pressor durante o exercício com oclusão vascular.

Tabela 1 Efeitos do reflexo pressor sobre os aspectos neuro-hormonais e cardiovasculares promovidos através do método oclusão vascular durante o exercício

\begin{tabular}{|cc|}
\hline Cardiovasculares & Neuro-hormonais \\
\hline $\mathrm{SNAS} \uparrow$ & Vasopressina (ADH) $\uparrow$ \\
\hline $\mathrm{SNAP} \downarrow$ & Catecolaminas $\uparrow$ \\
\hline $\mathrm{FC} \uparrow$ & $\mathrm{GH} \uparrow$ \\
\hline $\mathrm{PAS} \uparrow$ & Atividade da Renina $\uparrow$ \\
\hline $\mathrm{PAD} \uparrow$ & \\
\hline $\mathrm{RVPT} \uparrow$ & \\
\hline $\mathrm{DC} \uparrow$ & \\
\hline
\end{tabular}

$\mathrm{GH}=$ hormônio de crescimento, $\mathrm{ADH}$ = hormônio antidiurético, SNAS = sistema nervoso autonômico simpático, SNAP = sistema nervoso autonômico parassimpático, $\mathrm{FC}$ = frequência cardíaca, PAS = pressão arterial sistólica, PAD = pressão arterial diastólica, RVPT = resistência vascular periférica total, $\mathrm{DC}=$ débito cardíaco. 
Outro fator importante e que deve ser analisado em pesquisas futuras é a associação da resposta neuro-hormonal crônica promovida pelo método oclusão vascular e hipertrofia ventricular esquerda. Sabendo que a oclusão vascular pode gerar uma resposta exagerada do reflexo pressor, aumentando a atividade simpática, levando a perigosas elevações da função cardíaca, pressão arterial e resistência vascular periférica (SPRANGER et al., 2015).

A elevação crônica dos fatores hemodinâmicos (aumento da atividade cardíaca) e neuro-humorais (catecolaminas e sistema nervoso autonômico simpático) são responsáveis pelo remodelamento cardíaco patológico (GARCIA; INCERPI, 2008; MACIEL, 2011; VAN DER LAARSE). Aumentando a ativação de vias intracelulares associadas a hipertrofia ventricular concêntrica (GARCIA; INCERPI, 2008; MACIEL, 2011; VAN DER LAARSE). Dentre elas:

- Fosfolipase C (PLC);

- Fosfatitil inositol 3-quinase (PI3k);

- Quinase regulada por sinalização extracelular (ERK);

- Diacilgliceral (DAG);

- Calcineurina;

- Fator nuclear de ativação de célula T (NFAT);

- Alvo da rapamicina em mamíferos (mTOR);

A aplicação do método em usuários de esteroides anabolizantes deve ser levada também em consideração. Os mesmos apresentam alterações das estruturas cardíacas e funcionamento, função endotelial comprometida, apresentam perfil lipídico alterado, perfil trombótico elevado, aumento dos níveis de renina, aldosterona e também aumento da atividade nervosa simpática quando comparados com não usuários (ALVES et al., 2010; SCULTHORE et al., 2012). Seria prudente avaliar os riscos trombóticos em usuários de anabolizantes antes de aplicar o método oclusão vascular, pois o êmbolo pode deslocar para o cérebro, coração ou pulmões. Trazendo consequências desastrosas.

Outro fator importante é o uso de suplementos para fins de emagrecimento que possuem substâncias cardioaceleradoras e simpatomiméticas como efedrina, agonistas do receptores beta 2 , sibutramina, $\beta$-Methylphenethylamine, $\mathrm{N}$-alfa-dietilfeniletilamina, $\mathrm{N}$-caffeoyldopamine, $\mathrm{N}$-coumaroyldopamine e 1,3 dimetilamilamina que aumentam o risco para arritmias, infarto do miocárdio e acidente vascular encefálico (SCULTHORE et al., 2012; GELLER et al., 2015; COHEN, 2014). O uso do método oclusão vascular juntamente com essas substâncias também deve ser avaliado com muita cautela. 


\section{REFERÊNCIAS BIBLIOGRÁFICAS}

ALVES, M. J. et al. Abnormal neurovascular control in anabolic androgenic steroids users. Med Sci Sports Exerc, v. 42, n. 5, p. 865-871, 2010.

BELLI, Juliana Fernanda Canhadas et al. Comportamento do ergorreflexo na insuficiência cardíaca. Arquivos Brasileiros de Cardiologia, v. 97, n. 2, p. 171-178, 2011. COHEN, Pieter A. Hazards of hindsight-monitoring the safety of nutritional supplements. New England journal of medicine, v. 370, n. 14, p. 1277-1280, 2014. GARCIA, José Antonio Dias; INCERPI, Erika Kristina. Fatores e mecanismos envolvidos na hipertrofia ventricular esquerda e o papel anti-hipertrófico do óxido nítrico. Arquivos Brasileiros de Cardiologia, v. 90, n. 6, p. 443-450, 2008.

GELLER, Andrew I. et al. Emergency department visits for adverse events related to dietary supplements. New England Journal of Medicine, v. 373, n. 16, p. 1531-1540, 2015. IIDA, H. et al. Hemodynamic and autonomic nervous responses to the restriction of femoral blood flow by KAATSU. International Journal of KAATSU Training Research, v. 1, n. 2, p. 57-64, 2005.

IIDA, Haruko et al. Hemodynamic and neurohumoral responses to the restriction of femoral blood flow by KAATSU in healthy subjects. European journal of applied physiology, v. 100, n. 3, p. 275-285, 2007.

MACIEL, Benedito Carlos. A hipertrofia cardíaca na hipertensão arterial sistêmica: mecanismo compensatório e desencadeante de insuficiência cardíaca. Rev Bras Hipertens, v. 8, p. 409-13, 2001.

PIEPOLI, Massimo F. et al. Reduced peripheral skeletal muscle mass and abnormal reflex physiology in chronic heart failure. Circulation, v. 114, n. 2, p. 126-134, 2006.

PONIKOWSKI, Piotr P. et al. Muscle ergoreceptor overactivity reflects deterioration in clinical status and cardiorespiratory reflex control in chronic heart failure. Circulation, v. 104, n. 19, p. 2324-2330, 2001.

RUWHOF, Cindy; VAN DER LAARSE, Arnoud. Mechanical stress-induced cardiac hypertrophy: mechanisms and signal transduction pathways. Cardiovascular research, v. 47, n. 1, p. 23-37, 2000.

SCULTHORPE, Nicholas et al. Cardiovascular risk and androgenic anabolic steroids. British Journal of Cardiac Nursing, v. 7, n. 6, p. 266-275, 2012.

SHIMIZU, Ryosuke et al. Low-intensity resistance training with blood flow restriction improves vascular endothelial function and peripheral blood circulation in healthy elderly people. European journal of applied physiology, v. 116, n. 4, p. 749-757, 2016. SPRANGER, Marty D. et al. Blood flow restriction training and the exercise pressor reflex: a call for concern. American Journal of Physiology-Heart and Circulatory Physiology, v. 309, n. 9, p. H1440-H1452, 2015.

TAKANO, H. et al. Effects of low-intensity "KAATSU” resistance exercise on hemodynamic and growth hormone responses. International Journal of KAATSU Training Research, v. 1, n. 1, p. 13-18, 2005.

TAKANO, Haruhito et al. Hemodynamic and hormonal responses to a short-term low-intensity resistance exercise with the reduction of muscle blood flow. European Journal of applied Physiology, v. 95, n. 1, p. 65-73, 2005. 